\title{
Relative Efficacy and Safety of Prasugrel and Clopidogrel in Medically Managed Patients of High Risk Unstable Angina/Non-ST-Elevation Myocardial Infarction (UA/NSTEMI)
}

\author{
Authors \\ Vikas Chaudhary ${ }^{1}$, VK Katyal', Jyoti Kadian ${ }^{3}$, Kunal Mahajan ${ }^{4}$ \\ ${ }^{1}$ Assistant Professor, Department of Medicine, Pandit B. D. Sharma Post Graduate Institute of Medical \\ Sciences, Rohtak, Haryana (India). \\ ${ }^{2}$ Senior Professor, Department of Medicine, Pandit B. D. Sharma Post Graduate Institute of Medical \\ Sciences, Rohtak, Haryana (India). \\ ${ }^{3}$ Assistant Professor, Department of Medicine, Pandit B. D. Sharma Post Graduate Institute of Medical \\ Sciences, Rohtak, Haryana (India). \\ ${ }^{4}$ Senior Resident, Dept of Cardiology, Indira Gandhi Medical College, Shimla, Himachal Pradesh (India) \\ Corresponding Author \\ Dr Vikas Chaudhary \\ Email: drvikaschaudhary1@gmail.com
}

\begin{abstract}
Background: The patients of unstable angina / non-ST-elevation myocardial infarction (UA/NSTEMI) who are managed medically continue to have high risk of future adverse clinical outcomes and remain an understudied population of patients. The primary objective of this study was to compare the relative efficacy and safety of prasugrel and clopidogrel in medically managed patients of high risk UA/NSTEMI.

Methods: In this open label, prospective randomized study, 100 consecutive patients of high risk UA/NSTEMI were studied under two groups of 50 each. In one group, prasugrel was used with $40 \mathrm{mg}$ as loading dose \& $10 \mathrm{mg}$ as maintenance dose while in the second group, clopidogrel was used as $300 \mathrm{mg}$ loading dose followed by $75 \mathrm{mg}$ as maintenance dose for a study period of 1 month. The patients were observed for the primary and secondary efficacy end points during the hospital stay (0-5 days) and follow up at 30 days.

Results: The patients in the prasugrel group had lower incidence of major adverse cardiac events (MACE) as compared to clopidogrel group both during hospital stay (6 versus 16, $p<0.05$ ) and total number of adverse cardiac events observed during the study period of 1 month (10 versus 19, $p<0.05)$. Although statistically insignificant, due to small sample size of study population, prasugrel was demonstrated to have better efficacy in diabetic cohort of the patients. For safety concerns, no any episode of major bleeding was observed with either of the drugs and minor bleeding rate was comparable between the two groups.

Conclusion: Prasugrel resulted in greater benefit in reducing the ischemic events and improving the net outcome of ACS (high risk UA/NSTEMI) population in comparison to the standard clopidogrel therapy.
\end{abstract}




\section{INTRODUCTION}

The dual antiplatelet therapy for ACS with the combination of aspirin and clopidogrel reduces the cardiovascular mortality, myocardial infarction and also the stroke. ${ }^{1}$ It has been observed that $5 \%$ to $44 \%$ of the patients treated with clopidogrel show high platelet reactivity depending upon the clopidogrel dose and the patient population. ${ }^{2}$ It has also been suggested in the clinical studies that due to the reduced pharmacological response to clopidogrel, patients may be at increased risk of adverse clinical events including myocardial infarction and coronary stent thrombosis. $^{3-6}$

Both clopidogrel and prasugrel are prodrugs and belong to thienopyridines group of antiplatelets which act by antagonizing P2Y12 adenosine diphosphate (ADP) receptor, which is involved in platelet activation and stabilization of the platelet Aggregate. ${ }^{7-10}$ Prasugrel is a third-generation thienopyridine adenosine diphosphate P2Y12receptor antagonist that has been demonstrated to have more potency and less response variability than clopidogrel. ${ }^{11}$

There have been many studies, focusing on comparison of the efficacy and safety profiles of clopidogrel and prasugrel in patients of ACS who were managed with percutaneous intervention (PCI), in addition.

The TRITON TIMI-38 was the major trial, which demonstrated that in patients of ACS undergoing PCI, the patients who received prasugrel had lower rates of composite events of reinfarction, stent thrombosis and death compared with patients receiving clopidogrel. ${ }^{12,13}$

Patients with UA/NSTEMI acute coronary syndrome who are managed medically continue to have high risk of future cardiovascular complications and remain an understudied group of patients. TRILOGY ACS remains largest trial to date which compared efficacy and safety of prasugrel+aspirin with the current standard of care clopidogrel +aspirin in patients of UA/NSTEMI who were managed medically without revascularization. ${ }^{14}$ On a different note with the TRITON TIMI 38, the TRILOGY ACS showed no significant statistical difference between prasugrel and clopidogrel in preventing stroke, MI, or death during 2.5 years of follow up among UA/NSTEMI patients of $<75$ years of age.

In the present study we evaluated the relative efficacy and safety of the prasugrel and clopidogrel in medically managed patients of high risk UA/NSTEMI acute coronary syndrome.

\section{MATERIALS AND METHODS}

This study was carried out in 100 consecutive patients of high risk unstable angina/non-STsegment elevation myocardial infarction (UA/NSTEMI) admitted to CCU (cardiac care unit) at a tertiary care hospital over a period of 1.5 years. It was an open label prospective randomized trial where patients were allocated to the two groups of 50 each. The study enrolled both male and female patients of 18 to 75 years of age, who had ischemic/anginal symptoms of 10 minutes or more and presented within 72 hours of symptom onset. Patients were required to have a medical management strategy and without a revascularization plan for the index event. Other eligibility criteria included, patients having a TIMI risk score of 3 or more, a ST-segment depression of $1 \mathrm{~mm}$ or more, or having elevated cardiac biomarkers. Patients were excluded if they were unable to provide a written informed consent, were likely to undergo PCI/coronary intervention for the index event, had STEMI as the index event, had cardiogenic shock, refractory arrhythmia or NYHA Class IV CHF within previous 24 hours. Patients on hemodialysis, having history of bleeding tendencies, history of stroke/TIA and weighing less than $60 \mathrm{~kg}$ or more than 75 years of age were also excluded from the study.

Patients were studied under two groups of 50 each. The group A consisted of 50 patients in which the prasugrel was used and group B comprised of 50 patients, in which clopidogrel 
was used as a study drug. Group-A patients received 40mg of prasugrel as loading dose followed by $10 \mathrm{mg}$ daily as maintenance therapy, while Group-B patients were given clopidogrel $300 \mathrm{mg}$ as loading dose followed by $75 \mathrm{mg}$ daily as maintenance dose up to a study period of one month. After admission to CCU, detailed clinical examination of the patient was done and entered in patient's proforma. Immediately 12 lead ECG was done and blood samples were tested for blood urea, blood sugar, serum sodium, serum potassium, CPK MB - at 0, 12 and 24 hours, and troponin-I at 12-24 hours (by chemiluminescence assay). A chest $\mathrm{x}$-ray was done routinely at admission. The investigative profile, completed within 24 hours of admission to CCU included in addition, blood sugar (fasting/postprandial), complete lipid profile, SGOT/PT levels, and CPK MB levels. ECG was done morning/evening and was repeated in between if indicated. All patients received a standardized drug treatment for ACS on admission to $\mathrm{CCU}$ which included, aspirin, intravenous nitroglycerin, low molecular weight heparin and beta blockers. Patients having contraindication to beta blockers or developing side effects with beta blockers, received diltiazem, or other antianginals as alternative therapy. Glycoprotein IIb/IIIa inhibitors were used if indicated with defined reduction in doses of antiplatelets and LMWH as laid down. Other drugs like ACE inhibitors and antiarrythmics were used as and when indicated. Statins were given to all patients. Rosuvastatin $10 \mathrm{mg}$ or atorvastatin 20 mg was used, starting from the first day of admission to the first follow up.

Patients were studied under two protocols:-

-During hospital stay (0-5 days) and

-Follow up protocol (at 30 days)

The primary efficacy end points of the study were nonfatal MI, non fatal stroke major or minor bleeding episodes or rehospitalization due to cardiac ischemic events or death during the study from cardiovascular causes. Patients were reevaluated at 1 month under follow up protocol.
They were asked to report in between if any adverse coronary event developed. At one month they were examined thoroughly to find out any clinical or other evidence of any adverse clinical events such as ischemia with ECG changes including infarction, stroke, CHF, arrhythmia, bleeding episodes major or minor etc.

\section{Statistical Analysis}

At the end of the study, the data was analysed statistically by using student t-test (unpaired) for quantitative and Chi-square test for qualitative variables by using SPSS v. 17.0. A p value of $<$ 0.05 was considered as significant.

\section{RESULTS}

\section{Baseline Characteristics}

In this open label randomized study, 100 consecutive patients of acute coronary syndrome (High risk USA/NSTEMI) were studied under two groups of 50 patients each. Patients, who qualified for the inclusion criteria were taken. The baseline characteristics of both prasugrel and clopidogrel groups are shown in Table 1. The mean age of enrollment was about 55 years in both the groups. The baseline characteristics in relation to presence of risk factors were almost similar in both the groups. In Group-A (prasugrel), 5 patients were having history of diabetes, while in Group-B (clopidogrel), there were 6 diabetic patients. All other risk factors like family history, obesity, hypertension, history of IHD were also comparable between the two groups.

\section{Events during hospital stay}

During hospital stay all the major adverse cardiac events (MACE) were recorded. Numbers of deaths were equal in both the study groups (Table 2). One patient expired in Group-A and one in Group-B. Left ventricular failure (LVF) was observed in 1 patient in Group-A and 3 patients in Group-B. Recurrent angina occurred in 2 patients in Group-A and 8 patients in Group-B ( $\mathrm{p}<0.005)$. Ischemia with ECG changes was noted in 2 patients in Group-A and 4 in Group-B patients. No major or minor bleeding episodes occurred in 
both the study groups during stay at the hospital. Similarly, there were no arrhythmias or stroke observed in both the study groups. Thus during the hospital stay, it was observed that patients on prasugrel had lower incidence of adverse cardiac events like, LVF, recurrent angina, and ischemia with ECG changes, than the patients on clopidogrel therapy. When the total numbers of major adverse cardiac events observed during the hospital stay are compared, they are significantly more in the clopidogrel group than the prasugrel group (16 versus 6).

Events between the discharge from hospital and follow up at 30 days All patients were followed up to 30 days and were called in clinic after 30 days or in between if any adverse cardiac event occurred. In both the study groups within 30 days of follow up, 2 patients in Group-A had minor bleeding episode while 1 patient in Group-B suffered from minor bleeding episode (Table 3). Ischemia with ECG Changes occurred in 2 patients in Group-A and 2 patients in Group-B. There were no other significant events in both the study groups during the follow up of 30 days. Although statistically non-significant, the bleeding occurred more frequently in prasugrel group (4\%) than the clopidogrel group (2\%). A total of 4 MACE occurred in Group-A while 3 in the Group-B ( $p$ non significant). Therefore, unlike effect on MACE in the immediate period, after onset of UA/NSTEMI, prasugrel and clopidogrel were comparable in their efficacy up to 1 month after stabilization of ACS.

\section{Events- prasugrel versus clopidogrel by diabetic status}

There were a total of 11 patients of diabetes mellitus (known case as well as freshly diagnosed) in both the study groups, 5 cases in prasugrel group and 6 cases in the clopidogrel group (Table 4). One death occurred in Group-A and one death in Group-B. Both the deaths during the study occurred in diabetic patients, no mortality was observed in non-diabetic patients (Table 5). In diabetic cohort, bleeding occurred in one patient in Group-B while no bleeding occurred in GroupA patients. In non-diabetic cohort bleeding occurred in 2 patients in Group-A, while there was no bleeding episodes in Group-B. Thus, although hemorrhage occurred more frequently in prasugrel group than clopidogrel (2 cases versus 1), no bleeding was observed with prasugrel in the diabetic cohort in the study. In diabetic cohort, left ventricular failure (LVF) occurred in 1 patient in Group-B and none in Group-A. Recurrent angina occurred in 2 patients in group-B of diabetic cohort. Ischemia with ECG changes occurred in 1 patient in Group-A and 2 patients in Group-B in diabetic cohort. In diabetic as well as non-diabetic cohort the total numbers of MACE were more in clopidogrel group than the prasugrel group, but were statistically non- significant.

\section{Total events during the study}

When the total number of major adverse cardiac events observed during the study period in both the study groups were compared, there was a significant difference $(\mathrm{p}<0.05)$ between the two groups, in relation to the events during hospital stay (Table 6.). Considering the total MACE after discharge from hospital and upto first follow up (5-30 days), there occurred 4 events in prasugrel group and 3 events in the clopidogrel group. The number of MACE after discharge were more in prasugrel group (although statistically nonsignificant) because of more incidence of bleeding in prasugrel group. When total events during the study are concerned, a total of 10 events occurred in prasugrel group and a total of 19 events occurred in clopidogrel group ( $\mathrm{p}<0.05$, Table $6 \&$ Figure 1). 
Table 1. Baseline characteristics of study population.

\begin{tabular}{|l|c|c|c|}
\hline Characteristic & $\begin{array}{c}\text { Prasugrel } \\
(\mathrm{n}=50)\end{array}$ & $\begin{array}{c}\text { Clopidogrel } \\
(\mathrm{n}=50)\end{array}$ & $\mathrm{p}$-value \\
\hline Mean age \pm SD (years) & $55.98 \pm 10.81$ & $55.06 \pm 11.83$ & 0.685 \\
\hline Male & 37 & 36 & 0.821 \\
\hline Female & 13 & 14 & \\
\hline Heart rate (bpm) & $81.56 \pm 14.55$ & $82.32 \pm 15.26$ & 0.799 \\
\hline Systolic BP (mm Hg) & $124.84 \pm 21.95$ & $127.28 \pm 23.24$ & 0.590 \\
\hline Diastolic BP (mm Hg) & $76.92 \pm 10.04$ & $76.44 \pm 12.52$ & 0.832 \\
\hline Current smoker & 31 & 32 & 0.835 \\
\hline Diabetes mellitus & 5 & 6 & 0.749 \\
\hline Hypertension & 13 & 11 & 0.639 \\
\hline Family history & 4 & 5 & 0.726 \\
\hline Obesity (BMI>25) & 7 & 10 & 0.424 \\
\hline Past H/O IHD & 16 & 13 & 0.508 \\
\hline ST-segment depression & $38(76 \%)$ & $40(80 \%)$ & 0.629 \\
\hline T-wave inversion & $6(12 \%)$ & $8(16 \%)$ & 0.564 \\
\hline Deep symmetrical- T wave inversion & $3(6 \%)$ & $2(4 \%)$ & 0.646 \\
\hline Biphasic - T wave inversion & $3(6 \%)$ & $48 \%)$ & 0.695 \\
\hline TIMI RISK Score (Mean \pm SD) & $1.88 \pm 0.939$ & $1.8 \pm 0.925$ & 0.668 \\
\hline CPK-MB (0HR)(U/L) & $64.79 \pm 39.15$ & $63.52 \pm 43.79$ & 0.878 \\
\hline (12HR)(U/L) & $83.01 \pm 57.53$ & $71.609 \pm 50.230$ & 0.293 \\
\hline (24HR)(U/L) & $85.2 \pm 70.8$ & $87.691 \pm 59.811$ & 0.849 \\
\hline TROPONIN-I (ng/ml) & $7.12 \pm 10.95$ & $7.446 \pm 10.509$ & 0.881 \\
\hline Hemoglobin (g \%) & $11.248 \pm 1.527$ & $11.6 \pm 1.527$ & 0.239 \\
\hline Blood Urea (mg \%) & $34.94 \pm 18.122$ & $33.38 \pm 11.41$ & 0.607 \\
\hline Blood Sugar-F (mg \%) & $87.16 \pm 31.91$ & $87.0 \pm 24.62$ & 0.977 \\
\hline Blood Sugar PP (mg \%) & $117.4 \pm 37.92$ & $120.44 \pm 38.249$ & 0.690 \\
\hline Serum Uric Acid (mg \%) & $5.337 \pm 1.472$ & $4.924 \pm 1.856$ & 0.222 \\
\hline Serum Creatinine (mg \%) & $1.024 \pm 0.291$ & $0.994 \pm 0.266$ & 0.642 \\
\hline SGOT & $60.96 \pm 39.019$ & $55.42 \pm 32.279$ & 0.440 \\
\hline SGPT & $48.32 \pm 26.699$ & $41.64 \pm 22.176$ & 0.176 \\
\hline Triglycerides (mg \%) & $171.54 \pm 67.567$ & $147.82 \pm 67.322$ & 0.081 \\
\hline Cholesterol (mg \%) & $176.72 \pm 39.318$ & $162.18 \pm 38.004$ & 0.063 \\
\hline HDL (mg \%) & $39.52 \pm 7.382$ & $43.78 \pm 15.561$ & 0.083 \\
\hline LDL (mg \%) & $104.3 \pm 30.303$ & $93.50 \pm 27.582$ & 0.0653 \\
\hline VLDL (mg \%) & $31.54 \pm 14.178$ & $26.90 \pm 11.381$ & 0.074 \\
\hline
\end{tabular}

Table 2. Events during stay in the hospital

\begin{tabular}{|l|c|c|c|}
\hline Events & $\begin{array}{c}\text { Group-A } \\
(\mathrm{n}=50)\end{array}$ & $\begin{array}{c}\text { Group-B } \\
(\mathrm{n}=50)\end{array}$ & p-value \\
\hline Death & 1 & 1 & 1.00. \\
\hline Major Bleeding Episode & NIL & NIL & \\
\hline Minor Bleeding Episode & NIL & NIL & \\
\hline Arrythmia & NIL & NIL & \\
\hline Left Ventricular Failure(LVF) & 1 & 3 & 0.307 \\
\hline Recurrent Angina & 2 & 8 & $<0.05$ \\
\hline Stroke & NIL & NIL & \\
\hline Ischemia With ECG Changes & 2 & 4 & 0.399 \\
\hline Total MACE & 6 & 16 & $<0.05$ \\
\hline
\end{tabular}


Table 3. Events between discharge from hospital and follow up at 30 days

\begin{tabular}{|l|c|c|c|}
\hline EVENTS & $\begin{array}{c}\text { Group-A } \\
(\mathrm{n}=50)\end{array}$ & Group-B (n=50) & p-value \\
\hline Death & NIL & NIL & - \\
\hline Bleeding episode & 2 & 1 & 0.557 \\
\hline Stroke & NIL & NIL & - \\
\hline Ischemia with ECG changes & 2 & 2 & 1.00 \\
\hline CHF & NIL & NIL & - \\
\hline Total MACE & 4 & 3 & 0.695 \\
\hline
\end{tabular}

Table 4. Events- prasugrel versus clopidogrel in diabetic patients

\begin{tabular}{|l|c|c|c|}
\hline Subjects with Diabetes Mellitus (n=11) & Group-A (n=5) & Group-B $(\mathrm{n}=6)$ & $\mathrm{p}$-value \\
\hline Death & $1(20 \%)$ & $1(16.7 \%)$ & 1.00 \\
\hline Bleeding Episode & NIL & $1(16.7 \%)$ & 0.314 \\
\hline LVF & NIL & $1(16.7 \%)$ & 0.314 \\
\hline Recurrent Angina & NIL & $2(33.33 \%)$ & 0.153 \\
\hline Ischemia with ECG Changes & $1(20 \%)$ & $2(33.33 \%)$ & 0.557 \\
\hline Total MACE & 2 & 7 & 0.080 \\
\hline
\end{tabular}

Table 5. Events- prasugrel versus clopidogrel in non-diabetic patients

\begin{tabular}{|l|c|c|c|}
\hline $\begin{array}{l}\text { Subjects without Diabetes Mellitus } \\
(\mathrm{n}=89)\end{array}$ & $\begin{array}{c}\text { Group-A } \\
(\mathrm{n}=45)\end{array}$ & $\begin{array}{c}\text { Group-B } \\
(\mathrm{n}=44)\end{array}$ & p-value \\
\hline Death & NIL & NIL & - \\
\hline Bleeding Episode & 2 & NIL & 0.153 \\
\hline LVF & 1 & 2 & 0.557 \\
\hline Post MI Angina & 2 & 6 & 0.140 \\
\hline Ischemia With ECG Changes & 2 & 2 & 1.00 \\
\hline Total MACE & 7 & 10 & 0.424 \\
\hline
\end{tabular}

Table 6. Total events during study

\begin{tabular}{|l|c|c|c|}
\hline EVENTS & GROUP-A $(\mathrm{n}=50)$ & GROUP-B $(\mathrm{n}=50)$ & $\mathrm{p}$-value \\
\hline Hospital stay (0-5 days) & 6 & 16 & $<0.05$ \\
\hline 5-30 day & 4 & 3 & 0.695 \\
\hline Total events & 10 & 19 & $<0.05$ \\
\hline
\end{tabular}

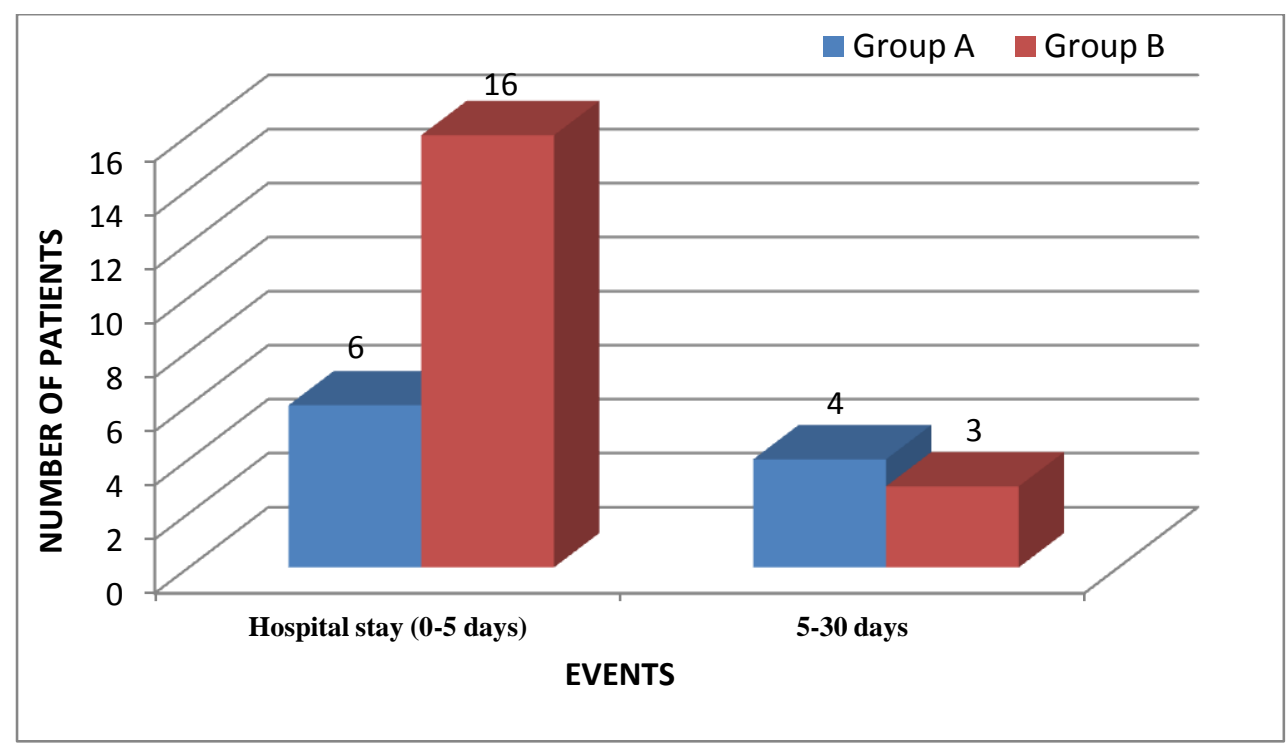

FIGURE 1: Total Events during Study 


\section{DISCUSSION}

The study was aimed to compare coronary and non coronary events upto 1 month of treatment with prasugrel and clopidogrel in a medically managed population of high risk UA/ NSTEMI patients. The safety profile of the two drugs was also compared. Prasugrel - a thienopyridine adenosine diphosphate receptor antagonist that provides higher and less variable levels of platelet inhibition than clopidogrel has demonstrated benefit when used to treat ACS patients undergoing percutaneous coronary intervention. ${ }^{12,15-18}$ However, the optimal approach to antiplatelet therapy for high risk medically managed NSTE ACS patient remains uncertain, as these patients have not been the focus of previous studies. Thus taking into account, the above consideration, we studied the efficacy and safety profile of the two drugs in a cohort of high risk of NSTE ACS population.

The present study of comparison between prasugrel and clopidogrel provides the findings which indicate significant advantage in preventing the various ischemic outcomes among patients of high risk unstable angina/ NSTEMI.

Like the major trials comparing prasugrel with clopidogrel in ACS patients undergoing PCI (TRITON-TIMI 38 TRIAL, the JUMBO-TIMI 26 Trial and the PRINCIPLE-TIMI 44 Trial), our study also showed the superiority of prasugrel over clopidogrel in reducing the ischemic events in the study population. The rate of bleeding was comparable in the prasugrel group with the clopidogrel group in our study. This could be due to small number of patients being enrolled in this study. Bleeding was not observed more frequently in prasugrel treated patients in present study unlike other studies. ${ }^{12,15}$ Two patients (4\%) in the prasugrel group suffered from minor bleeding episode while one patient (2\%) had minor bleeding episode in clopidogrel group.

In the TRITON TIMI 38 trial, the relative risk of recurrent ischemic events was reduced by $30 \%$ in the prasugrel group among patients treated with
PCI. Major bleeding occurred in $2.4 \%$ of patients receiving prasugrel and in $1.8 \%$ of patients receiving clopidogrel in the TRITON trial (hazard ratio, $1.32 ; 95 \% \mathrm{CI}, 1.03$ to $1.68 ; \mathrm{P}=0.03$ ). The prasugrel group also had the higher rate of lifethreatening bleeding $(1.4 \%$ vs. $0.9 \% ; \mathrm{P}=0.01)$, nonfatal bleeding (1.1\% vs. $0.9 \%$; hazard ratio, $1.25 ; \mathrm{P} 0.23)$ and fatal bleeding $(0.4 \%$ vs. $0.1 \%$; $\mathrm{P}$ $=0.002$ ).

In the TRILOGY ACS, the largest trial till date of ACS patients managed medically without revascularization, the prasugrel have not shown any significant difference from clopidogrel during 2.5 years of follow up among patients of $<75$ years of age. ${ }^{14} \mathrm{~A}$ time dependent trend for benefit was observed after 1 year in TRILOGY ACS patients such that fewer total recurrent ischemic events occurred with the prasugrel, particularly after 1 year. No statistical difference was observed in major life threatening or fatal bleeding with prasugrel versus clopidogrel. A substudy of TRILOGY ACS on platelet function showed a lower frequency of high on-treatment platelet reactivity with prasugrel than with clopidogrel but no independent association between on-treatment platelet reactivity and ischemic outcomes. ${ }^{19}$

In our study second line anti-anginals like diltiazem, nicorandil were used more frequently in clopidogrel patients. Thus more usage of the second line anti-anginals in clopidogrel patients over prasugrel patients, indicates more incidence of residual ischemia in clopidogrel group of patients. The use of additional anti-anginal drugs in the present study was necessary because of burden of myocardial ischaemia in the patients unwilling for coronary interventions.

Diabetic patients in the study had higher incidence of the adverse coronary events than the non diabetic patients. In the diabetic cohort, the number of adverse cardiac events were more in the clopidogrel group (7 versus 2), although not statistically significant due to small sample of study population. 
In the TRITON TIMI 38 trial also, the prasugrel demonstrated higher efficacy in diabetic patients. Although prasugrel had superior results in both diabetic and non diabetic patients, but a greater relative benefit was demonstrated in patients with diabetes as compared to non diabetic patients. The data from our study as well as the TRITON TIMI 38 supports the observations of higher platelet reactivity and requirement of intensive antiplatelet therapy in diabetic patients. $^{20-23}$ The non enzymatic glycation of the proteins on the surface of platelets, induced by the hyperglycemia leads to the decreased membrane fluidity and consequent increase in the propensity of the platelets to activate. ${ }^{24-26}$ A recent meta-analysis by Rossington et $\mathrm{al}^{27}$ shows that the addition of a P2Y12 inhibitor is superior to placebo, with a trend favouring the use of prasugrel in patients with diabetes with ACS, particularly those undergoing PCI.

Thus it is apparent from the study that prasugrel, a potent oral P2Y12 inhibitor, is more effective at preventing ischemic events than is the inhibition offered by the standard dose of clopidogrel. Use of prasugrel was associated with less number of major adverse cardiac events (MACE) than the patients who were on clopidogrel. Although for the individual adverse cardiac events, there was no statistically significant difference, but when the total MACE observed during the study are compared, they were significantly less in patients on prasugrel therapy. Diabetes mellitus was associated with the more adverse coronary outcomes in patients of ACS than the non diabetic ACS patients in the present study. This conforms to other previous studies. Prasugrel use in diabetic patients was of greater benefit in comparison to the standard clopidogrel therapy, than in the non diabetic patients in present study. Safety of prasugrel in present study was identical to clopidogrel possibly because of small size of sample.

\section{CONCLUSION}

In this analysis from present study, we have demonstrated that in comparison to the standard clopidogrel therapy, prasugrel resulted in greater benefit in reducing the ischemic events and improving the net outcome of ACS (high risk UA/NSTEMI) population. Prasugrel was specially more effective in high risk ACS patients with diabetes mellitus. Although event rate was less in prasugrel group than the clopidogrel group of patients, this did not reach statistical significance, in non-diabetic population; possibly due to small number of patients in the study group. A large study group is essential to detect exact benefit with either drug in the Indian or subcontinent population.

\section{REFERENCES}

1. Fox KA, Mehta SR, Peters R. Benefits and risks of the combination of clopidogrel and aspirin in patients undergoing surgical revascularization for non- ST-elevation acute coronary syndrome: the clopidogrel in unstable angina to prevent recurrent ischemic events (CURE) trial. Circulation 2004;110:1202-8.

2. Gurbel PA, Tantry US. Clopidogrel resistance?. Thrombos Res 2007;120:31121.

3. Buonamici P, Marcucci R, Migliorini A. Impact of platelet reactivity after clopidogrel administration on drug-eluting stent thrombosis. J Am Coll Cardiol 2007;49:2312-7.

4. Gurbel PA, Bliden KP, Samara W. Clopidogrel effect on platelet reactivity in patients with stent thrombosis: results of the CREST Study. J Am Coll Cardiol 2005;46:1827-32.

5. Hochholzer W, Trenk D, Bestehorn HP. Impact of the degree of peri-interventional platelet inhibition after loading with clopidogrel on early clinical outcome of 
elective coronary stent placement. J Am Coll Cardiol 2006;48:1742-50.

6. Matetzky S, Shenkman B, Guetta V. Clopidogrel resistance is associated with increased risk of recurrent atherothrombotic events in patients with acute myocardial infarction. Circulation 2004; 109:3171-5.

7. Savi P, Herbert JM. Clopidogrel and ticlopidine: P2Y12 adenosine diphosphatereceptor antagonists for the prevention of atherothrombosis. Semin Thromb Hemost 2005;31:174-83.

8. Sugidachi A, Asai F, Ogawa T. The in vivo pharmacological profile of CS-747, a novel antiplatelet agent with platelet ADP receptor antagonist properties. $\mathrm{Br} \mathrm{J}$ Pharmacol 2000;129:1439-46.

9. Goto S, Tamura N, Ishida H. Dependence of platelet thrombus stability on sustained glycoprotein IIb/IIIa activation through adenosine 5-diphosphate receptor stimulation and cyclic calcium signaling. J Am Coll Cardiol 2006;47:155-62.

10. Cattaneo M. The platelet P2Y receptors as targets for new antithrombotic drugs. J Thromb Haemost 2003;1:1133-5.

11. Jakubowski JA, Winters KJ, Naganuma H. Prasugrel: a novel thienopyridine antiplatelet agent. A review of preclinical and clinical studies and the mechanistic basis for its distinct antiplatelet profile. Cardiovasc Drug Rev 2007;25:357-74.

12. Wiviott SD, Braunwald E, McCabe $\mathrm{CH}$. Prasugrel versus clopidogrel in patients with acute coronary syndromes. N Engl J Med 2007;357:2001-15.

13. Morrow DA, Wiviott SD, White HD. Effect of the novel thienopyridine prasugrel compared with clopidogrel on spontaneous and procedural myocardial infarction in TRITON-TIMI 38: an application of the classification system from the universal definition of myocardial infarction. Circulation 2009;119:2758-64.

14. Roe MT, Armstrong PW, Fox KA. Prasugrel versus clopidogrel for acute coronary syndromes without revascularization. N Engl J Med 2012;367:1297-309.

15. Zeymer U, Hochadel M, Lauer B, Kaul N, Wohrle J, Andresen D, et al. Use, efficacy and safety of prasugrel in patients with ST segment elevation myocardial infarction scheduled for primary percutaneous coronary intervention in clinical practice. Results of the prospective ATACSregistry. Int J Cardiol 2015;184:122-7.

16. Dasbiswas A, Rao MS, Babu PR. A comparative evaluation of prasugrel and clopidogrel in patients with acute coronary syndrome undergoing percutaneous coronary intervention. J Assos Phys India 2013;61:28-40.

17. Wiviott SD, Trenk D, Frelinger AL. Prasugrel compared with high loading-and maintenance-dose clopidogrel in patients with planned percutaneous coronaruy intervention: the Prasugrel in Comparison to Clopidogrel for Inhibition of Platelet Aggregation- Thrombolysis in Myocardial Infarction 44 trial. Circulation 2007;116:2923-32.

18. Wiviott SD, Antman ME, Winters KJ. Randomized comparison of prasugrel (CS747, LY640315), a novel thienopyridine P2Y12 antagonist, with clopidogrel in percutaneous coronary intervention: results of the Joint Utilization of Medications to Block Platelets Optimally (JUMBO)-TIMI 26 trial. Circulation 2005;111:3366-73.

19. Gurbel PA, Erlinge D, Ohman EM. Platelet function during extended prasugrel and clopidogrel therapy for patients with ACS treated without revascularization: the TRILOGY ACS Platelet Function Substudy. JAMA 2012;308:1785-94. 
20. Wiviott SD, Braunwald E, Angiolillo DJ. Greater clinical benefit of more intensive oral antiplatelet therapy with prasugrel in patients with diabetes mellitus in the trial to assess improvement in therapeutic outcomes by optimizing platelet inhibition with prasugrel-Thrombolysis in Myocardial Infarction 38. Circulation 2008;118:1626-36.

21. Angiolillo DJ, Fernandez-Ortiz A, Bernardo E. Platelet function profiles in patients with type 2 diabetes and coronary artery disease on combined aspirin and clopidogrel treatment. Diabetes 2005;54:2430-35.

22. Kim JH, Bae HY, Kim SY. Clinical marker of platelet hyperreactivity in diabetes mellitus. Diab Metabol J 2013;37:423-8.

23. Marso SP, Lincoff AM, Ellis SG. Optimizing the percutaneous interventional outcomes for patients with diabetes mellitus: results of the EPISTENT (Evaluation of Platelet IIb/IIIa Inhibitor for Stenting Trial) diabetic substudy. Circulation 1999; 100:2477-84.

24. Winocour PD, Watala C, KinloughRathbone RL: Membrane fluidity is related to the extent of glycation of proteins, but not to alterations in the cholesterol to phospholipid molar ratio in isolated platelet membranes from diabetic and control subjects. Thromb Haemost 1992;67:567-71.

25. Winocour PD, Watala C, Perry DW, Kinlough-Rathbone RL. Decreased platelet membrane fluidity due to glycation or acetylation of membrane proteins. Thrombosis and Haemostasis 1992;68:577-82.

26. Watala C, Boncer M, Golański J. Platelet membrane lipid fluidity and intraplatelet calcium mobilization in type 2 diabetes mellitus. European J Haematol 1998;61:319-26.

27. Rossington JA, Brown OI, Hoye A. Systematic review and meta-analysis of optimal P2 $\mathrm{Y}_{12}$ blockade in dual antiplatelet therapy for patients with diabetes with acute coronary syndrome. Open Heart 2016;3:e000296. 